\title{
Relationship between time-varying status of reflux esophagitis and Helicobacter pylori and progression to long-segment Barrett's esophagus: time-dependent Cox proportional-hazards analysis
}

Genki Usui ${ }^{1 *}$ (O), Tomohiro Shinozaki ${ }^{2}$, Toyohisa Jinno $^{3}$, Kazutoshi Fujibayashi $^{4}$, Teppei Morikawa', Toshiaki Gunji and Nobuyuki Matsuhashi ${ }^{5}$

\begin{abstract}
Background: Reflux esophagitis (RE) and absence of Helicobacter pylori (non-H. pylori) are considered to be associated with the progression to long-segment Barrett's esophagus (LSBE). However, it is difficult to assess this association because RE and $H$. pylori status can change during follow-up. Additionally, the association between $H$. pylori eradication and LSBE remains unclear.

Methods: A total of 11,493 asymptomatic Japanese subjects who underwent medical check-ups and were endoscopically diagnosed with short-segment Barrett's esophagus (SSBE) between May 2006 and December 2015 were enrolled. The hazards of progression to LSBE were compared between time-varying RE and H. pylori infection/ eradication by time-dependent multivariable Cox proportional hazards models.

Results: A total of 7637 subjects who underwent additional medical check-ups after being diagnosed with endoscopic SSBE were analyzed. Subjects with RE and without current/past H. pylori infection were strongly associated with a higher rate of progression to LSBE (adjusted hazard ratio [HR]: 7.17, 95\% confidence interval [CI]: 2.48-20.73, $p<0.001$ for RE and non-H. pylori vs. non-RE and H. pylori groups). Subjects with H. pylori had a lower rate of progression to LSBE (adjusted HR: 0.48, 95\% Cl: 0.22-1.07, $p=0.07$ for $H$. pylori vs. non-H. pylori). Hazards of progression to LSBE were still lower in the $H$. pylori eradication group than that of the non-H. pylori group (adjusted HR: $0.51,95 \%$ Cl: $0.18-1.46, p=0.21$ ).
\end{abstract}

Conclusions: RE and non-H. pylori were associated with the progression to LSBE, considering the changes in exposures. H. pylori infection was associated with the prevention of the development of LSBE irrespective of RE. The environment preventive of the development of LSBE persists for at least a few years after $\mathrm{H}$. pylori eradication.

Keywords: Helicobacter pylori, Eradication, Reflux esophagitis, Barrett's esophagus, SSBE, LSBE, Endoscopy, Elongation, Time-dependent, Time-varying

\footnotetext{
*Correspondence: gusui-tky@umin.ac.jp

'Department of Diagnostic Pathology, NTT Medical Center Tokyo, 5-9-22

Higashi-gotanda, Shinagawa-ku, Tokyo 141-8625, Japan

Full list of author information is available at the end of the article
}

(C) The Author(s). 2020 Open Access This article is licensed under a Creative Commons Attribution 4.0 International License, which permits use, sharing, adaptation, distribution and reproduction in any medium or format, as long as you give appropriate credit to the original author(s) and the source, provide a link to the Creative Commons licence, and indicate if changes were made. The images or other third party material in this article are included in the article's Creative Commons licence, unless indicated otherwise in a credit line to the material. If material is not included in the article's Creative Commons licence and your intended use is not permitted by statutory regulation or exceeds the permitted use, you will need to obtain permission directly from the copyright holder. To view a copy of this licence, visit http://creativecommons.org/licenses/by/4.0/. The Creative Commons Public Domain Dedication waiver (http://creativecommons.org/publicdomain/zero/1.0/) applies to the data made available in this article, unless otherwise stated in a credit line to the data. 


\section{Background}

Barrett's esophagus (BE) is known as a premalignant lesion of esophageal adenocarcinoma (EAC) [1-3]. BE is classified into short-segment $\mathrm{BE}(<3 \mathrm{~cm}) \quad(\mathrm{SSBE})$ and long-segment $\mathrm{BE}(\geqq 3 \mathrm{~cm})$ (LSBE) based on the segment lengths of esophageal mucosa with columnar metaplasia [4]. LSBE, a higher-risk condition for the development of EAC than SSBE, is common in Western countries [5]. On the other hand, the lengths of most BEs in Asia are less than $3 \mathrm{~cm}[6,7]$. In Japan, more patients are endoscopically diagnosed with SSBE, some of which are known to have different risk factors than those with LSBE [8]. It is clinically essential to investigate the frequency and clinical characteristics of endoscopic SSBE in developing into LSBE or EAC. However, the natural history of endoscopic SSBE elongation remains unknown.

Reflux esophagitis (RE) and absence of Helicobacter pylori (non- $H$. pylori) are considered to be associated with SSBE elongation to LSBE [9]. H. pylori infection causes gastric atrophy and decreases gastric acid secretion, leading to a decreased prevalence of RE and BE [10, 11]. Past studies showed that $H$. pylori infection suppressed the elongation of SSBE (odds ratio [OR]: 0.71, 95\% confidence interval [CI]: 0.44-1.15) [9]. However, it is difficult to accurately assess these associations because some RE subjects start or stop taking antacids, and some $H$. pylori infections are eradicated during follow-up. There have been no studies that investigate the associations referring to changes in RE and $H$. pylori status.

The association between $H$. pylori eradication and progression to LSBE remains unclear. $H$. pylori eradication is a useful treatment for reducing the risk of developing gastric cancer [12, 13]. However, some studies reported that $H$. pylori eradication increases gastric acid secretion, leading to an increased prevalence of RE [14]. Therefore, H. pylori eradication may also promote BE [15], which has not yet been confirmed.

There are two severe challenges in accurately estimating the association between RE and $H$. pylori status and progression to LSBE. First, it is challenging to collect clinical data several times over time. In particular, the assessment of RE and $H$. pylori status requires endoscopy and $H$. pylori tests, such as the serum anti-H. pylori IgG antibody test, which are not routinely conducted. The second is the analysis of time-varying exposures. In Cox proportional hazards regression models, exposures are usually fixed at baseline. However, especially in observational studies with long follow-up periods, exposures can often change during follow-up. Cox models with covariates fixed at baseline may mislead the true association by misclassifying exposures during the followup period.

Therefore, we conducted a retrospective cohort study aimed at investigating the association between RE and
H. pylori status and progression to LSBE using medical check-up data collected over time. We used timedependent covariates in the Cox models, which may estimate the accurate impact of RE or $H$. pylori infection and eradication on the progression to LSBE.

\section{Methods \\ Study population and design}

A total of 11,493 asymptomatic Japanese subjects who underwent the Early Disease Detection and Prevention program at NTT Medical Center Tokyo in Japan and who were endoscopically diagnosed with SSBE from May 2006 to December 2015 were enrolled. In this hospital, the Early Disease Detection and Prevention program is conducted with asymptotic subjects $[8,16,17]$. This program includes medical interviews about alcohol consumption, smoking habits, medical and treatment histories, physical and physiological examinations, blood tests including serum anti-H. pylori IgG antibody test, and esophagogastroduodenoscopy screening. Subjects with a history of esophagectomy or gastrectomy were excluded from the analysis. Subjects who did not undergo an additional program after being diagnosed with SSBE were also excluded. Our study protocol was approved by the institutional ethics committee.

\section{Data collection}

Endoscopy specialists who did not know the subjects' information performed esophagogastroduodenoscopy. The endoscopic diagnosis of $\mathrm{RE}$ and $\mathrm{BE}$ was conducted according to the Los Angeles classification system [18, 19], and Prague C \& M Criteria [20]. In accordance with a previous multicenter prospective cohort study in Japan, the site of the esophagogastric junction was defined in our study as the proximal end of the mucosal folds continuous from the stomach or distal ends of the palisade vessels [21]. Endoscopically diagnosed BE is called endoscopic BE, columnar-lined esophagus or endoscopically suspected esophageal metaplasia [22-25]. When the distance between the circumferential extension of the gastric mucosal fold and the proximal edge was $1-3 \mathrm{~cm}$ or exceeded $3 \mathrm{~cm}$, it was defined as an endoscopic SSBE or LSBE, respectively. After endoscopists conducted the endoscopic examinations, the most experienced endoscopist (N.M.) reviewed key images of all examinations. He conducted weekly endoscopic image reviews (3-12 key images per subject) throughout the study period to confirm the accurate diagnosis.

$H$. pylori infection was defined as $H$. pylori seropositivity and the presence of endoscopic atrophic gastritis (AG). Serological $H$. pylori status was assessed by using an enzyme-linked immunosorbent assay (Eiken Chemical, Tokyo, Japan). The seropositive antibody titer threshold for $H$. pylori infection was set at $10 \mathrm{U} / \mathrm{ml}$. Its 
sensitivity and specificity were over $85 \%[26,27]$. $H$. pylori seronegative and endoscopically AG negative subjects were categorized as non- $H$. pylori infection, and the others were categorized as $H$. pylori infection. Subjects with $H$. pylori infection were classified into current $H$. pylori infection and $H$. pylori eradication groups. These categories were updated at each subject's visit through the follow-up period.

Smoking habits were assessed using pack-years (packs of cigarettes per day multiplied by smoking years). Subjects were categorized into four groups according to the amount of alcohol consumption per week: nondrinker $(<40 \mathrm{~g} /$ week), light drinker (40-140 g/week), moderate drinker (140-280 g/week), and heavy drinker (> $280 \mathrm{~g} /$ week) $[8,16]$.

\section{Statistical analysis}

The subjects were categorized into four groups according to the combinations of RE and H. pylori status (non$H$. pylori or $H$. pylori infection). The probability of progressing to LSBE according to RE and $H$. pylori status at baseline was estimated using the Kaplan-Meier method. This analysis did not take into account both the change in RE and $H$. pylori status. In subsequent analyses, the following information was updated in a time-dependent manner: age, smoking, RE, medication (proton pump inhibitor (PPI) or histamine H2-receptor antagonist (H2RA)), and H. pylori status. The rate of progression to LSBE for each time-varying RE and $H$. pylori status was calculated using the person-years method and compared between the RE and H. pylori statuses through hazard ratios (HRs) estimated by multivariable Cox models with time-dependent covariates. In these models, the covariates, including RE and $H$. pylori status, were updated at a medical check-up. If a subject progressed to LSBE after a status change, this information contributes to event counts in the new status, while information about not progressing to LSBE by the progression time contributes to both the previous and the new statuses depending on her/his observed time. The association between H. pylori status (current infection or eradication) and progression to LSBE was also assessed by time-dependent Cox models. In model 1 , subjects were categorized into two groups: non- $H$. pylori infection and $H$. pylori infection groups. In model 2 , subjects were categorized into three groups: non-H. pylori infection, current $H$. pylori infection, and $H$. pylori eradication groups. Because the convergence of parameter estimates was not achieved in the initial Cox model that fully adjusted for possible risk factors, we carefully checked the convergence and estimates for every combination of covariates adjusted. Accordingly, we selected sex, age, smoking, hiatal hernia, RE, and medication (PPI or H2RA) to adjust for, as we found alcohol consumption did not predict the outcome in either univariable or multivariable models.
We conducted the statistical analysis by using SAS 9.4 (SAS Institute, Cary, NC) and EZR (Saitama Medical Center, Jichi Medical University, Saitama, Japan), which is a graphic user interface for $\mathrm{R}$ (The $\mathrm{R}$ Foundation for statistical computing, Vienna, Austria). EZR is an enhanced version of the $\mathrm{R}$ commander with a statistical function [28].

\section{Results}

Among the 11,493 subjects who were diagnosed with endoscopic SSBE between May 2006 and December 2015, 192 subjects with a history of esophagectomy or gastrectomy were excluded. Among the remaining 11,301 subjects, 7637 subjects who underwent additional health checks by November 2018 were included in the analysis.

The baseline characteristics of the subjects with endoscopic SSBE are presented in Supplemental Table 1. At baseline, a total of 4067 subjects (53.3\%) were negative for $H$. pylori infection, 2732 subjects $(35.8 \%)$ were currently positive for $H$. pylori infection, and 819 subjects (10.7\%) had a history of successful $H$. pylori eradication. A total of 3176 subjects (41.6\%) were negative for $\mathrm{RE}$ and positive for $H$. pylori infection, 3020 subjects (39.5\%) were negative for RE and H. pylori infection, 375 subjects (4.9\%) were positive for RE and $H$. pylori infection, and 1047 subjects (13.7\%) were positive for RE and negative for $H$. pylori infection. The characteristics of the subjects categorized according to baseline RE and $H$. pylori infection status are presented in Table 1.

The median follow-up period was 4.0 years. During the follow-up period, 34 subjects showed progression to LSBE. The Kaplan-Meier curves show the probability of not progressing to $\mathrm{LSBE}$, according to RE and $H$. pylori status at baseline (Fig. 1). These curves suggested that the probability of progression to LSBE was lower in subjects without RE and with $H$. pylori infection than in the other groups. However, this analysis did not consider changes in RE or $H$. pylori status. In subsequent analyses, the covariates were time-dependently updated. Of the 7637 subjects with endoscopic SSBE, none had lowor high-grade dysplasia during the study.

During the follow-up period, the rate of progression to LSBE was 1.0 per 1000 person-years. The progression rate for each time-varying $\mathrm{RE}$ and $H$. pylori status was as follows: 0.4 (non-RE and $H$. pylori infection), 0.9 (non$\mathrm{RE}$ and non-H. pylori infection), 2.2 (RE and H. pylori infection), and 2.8 per 1000 person-years (RE and nonH. pylori infection) (Table 2).

Table 3 shows HRs of time-varying RE and H. pylori status for the progression to LSBE in the multivariable time-dependent Cox models. RE and non- $H$. pylori group and RE and H. pylori group were strongly associated with a higher rate of progression to LSBE (adjusted HR: 7.17, 95\% CI: 2.48-20.73, $p<0.001$ for RE and non- 
Table 1 Differences in various parameters by RE and H. pylori status pattern at baseline

\begin{tabular}{|c|c|c|c|c|}
\hline & $\begin{array}{l}\text { RE }(-) / H . \text { pylori }(+) \\
(n=3176)\end{array}$ & $\begin{array}{l}\mathrm{RE}(-) / H \text {. pylori }(-) \\
(n=3020)\end{array}$ & $\begin{array}{l}\text { RE(+)/H. pylori(+) } \\
(n=375)\end{array}$ & $\begin{array}{l}\mathrm{RE}(+) / H \text {. pylori(-) } \\
(n=1047)\end{array}$ \\
\hline Age (years) (mean (SD)) & $54.5(9.6)$ & $47.3(10.0)$ & $52.9(8.7)$ & $47.7(9.4)$ \\
\hline Sex, men (\%) & $2618(82.4)$ & $2351(77.8)$ & $353(94.1)$ & $936(89.4)$ \\
\hline Smoking (pack-years) (mean \pm SD) & $14.8(17.3)$ & $10.3(14.9)$ & $20.0(18.9)$ & $12.4(15.0)$ \\
\hline \multicolumn{5}{|l|}{ Alcohol consumption } \\
\hline Nondrinker (< 40 g/week) (\%) & $1185(37.4)$ & $1210(40.1)$ & $110(29.3)$ & $391(37.3)$ \\
\hline Light-drinker (40-140 g/week) (\%) & $848(26.7)$ & $846(28.0)$ & $90(24.0)$ & $266(25.4)$ \\
\hline Moderate-drinker (140-280 g/week) (\%) & $615(19.4)$ & $558(18.5)$ & $83(22.1)$ & $217(20.7)$ \\
\hline Heavy-drinker ( $\geq 280 \mathrm{~g} /$ week) (\%) & $524(16.5)$ & $406(13.4)$ & $92(24.5)$ & $173(16.5)$ \\
\hline Hiatal hernia (\% positive) & $642(20.2)$ & $939(31.1)$ & $138(36.8)$ & $425(40.6)$ \\
\hline PPI or H2RA (\% positive) & $77(2.4)$ & $76(2.5)$ & $8(2.1)$ & $15(1.4)$ \\
\hline
\end{tabular}

RE Reflux esophagitis; H. pylori Helicobacter pylori; SD Standard deviation; PPI Proton pump inhibitor; H2RA Histamine H2-receptor antagonist

H. pylori vs. non-RE and $H$. pylori group; adjusted HR: 4.57, 95\% CI: $1.26-16.63, p=0.02$ for RE and H. pylori vs. non-RE and $H$. pylori group). Non-RE and non- $H$. pylori infection groups were also associated with a higher rate of progression to LSBE but were imprecisely estimated due to limited event numbers (adjusted HR:
2.45, 95\% CI: 0.86-7.00, $p=0.09$ for non-RE and non- $H$. pylori vs. non-RE and $H$. pylori groups). In the multivariable Cox models with fixed covariates, the HR of RE and non- $H$. pylori for the progression to LSBE was attenuated (adjusted HR: 5.59, 95\% CI: 2.04-15.36, $p<0.001$ for RE and non- $H$. pylori vs. non-RE and $H$. pylori

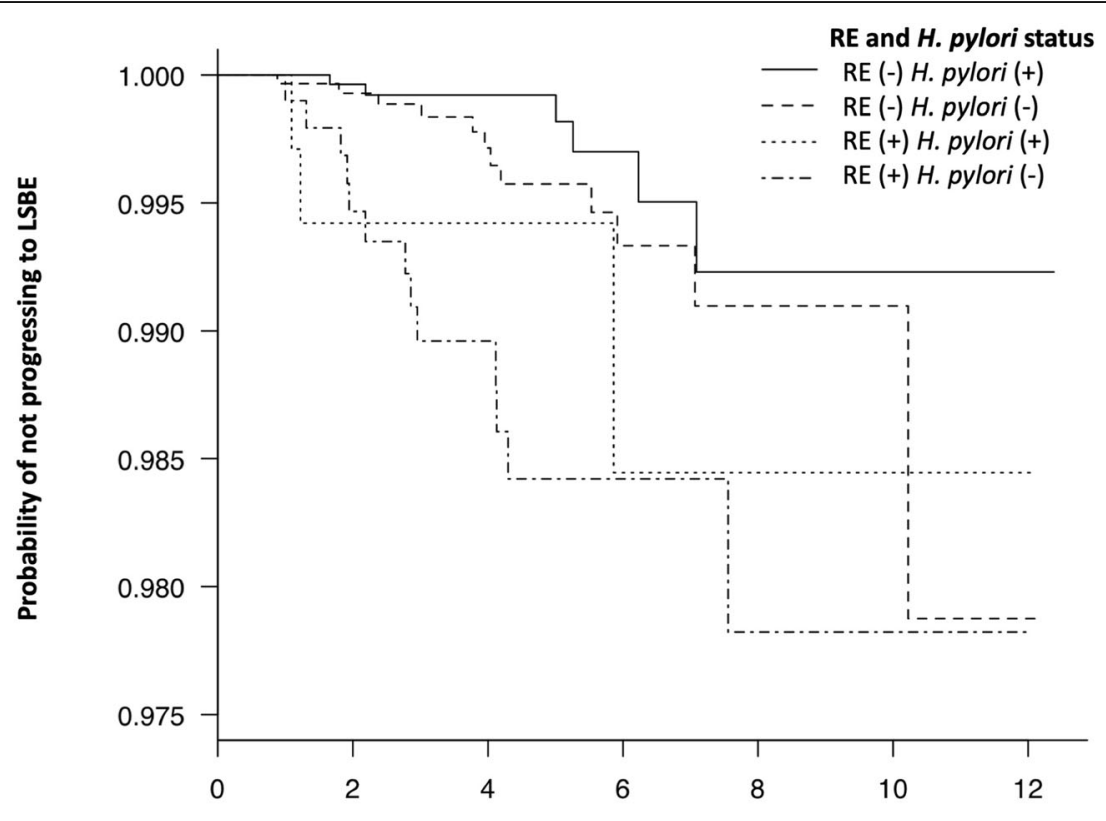

Years since diagnosis of endoscopic SSBE

$\begin{array}{lrrrrrrr}\text { No. at Risk } & & & & & & & \\ \text { RE (-) H. pylori (+) } & 3,176 & 2,535 & 1,466 & 597 & 223 & 74 & 3 \\ \text { RE (-) H. pylori (-) } & 3,020 & 2,518 & 1,516 & 691 & 267 & 100 & 5 \\ \text { RE (+) H. pylori (+) } & 375 & 305 & 187 & 91 & 30 & 10 & 1 \\ \text { RE (+) H. pylori (-) } & 1,047 & 884 & 593 & 304 & 130 & 60 & 1\end{array}$

Fig. 1 Kaplan-Meier estimates of the probability of not progressing to LSBE, according to RE and H. pylori status. Curves are shown for the probability of not progressing to LSBE according to RE and H. pylori status 
Table 2 The rate of progression to LSBE for each time-varying RE and $H$. pylori status

\begin{tabular}{llll}
\hline Time-varying RE and H. pylori status & Person-years & Elongation of SSBE to LSBE & $\begin{array}{l}\text { Rate } \\
\text { (per 1000 person-years) }\end{array}$ \\
\hline $\mathrm{RE}(-) / H$. pylori (+) & 12,889 & 5 & 0.4 \\
$\mathrm{RE}(-) /$ H. pylori (-) & 13,571 & 12 & 0.9 \\
$\mathrm{RE}(+) /$ H. pylori (+) & 1829 & 4 & 2.2 \\
$\mathrm{RE}(+) /$ /H. pylori (-) & 4710 & 13 & 2.8 \\
\hline
\end{tabular}

SSBE Short-segment Barrett's esophagus; LSBE Long-segment Barrett's esophagus; RE Reflux esophagitis; H. pylori Helicobacter pylori

groups) (Supplemental Table 2). In addition, the HR of smoking for the progression to LSBE was lower in Cox models by baseline time-fixed covariate (adjusted HR (per 100 pack-years): 1.64, 95\% CI: 0.29-9.11, $p=0.57)$ than that in the time-dependent Cox model (adjusted HR (per 100 pack-years): 3.66, 95\% CI: $1.09-12.29, p=0.04$ ).

Table 4 shows HRs of time-varying $H$. pylori status for the progression to LSBE in the multivariable timedependent Cox models. Model 1 showed an adjusted HR of 0.48 (95\% CI: $0.22-1.07, p=0.07$ for $H$. pylori vs. non-H. pylori), which suggests a decrease in the rate of progression to LSBE by $H$. pylori infection. In model 2, the hazards of progression to LSBE were still lower than those of non- $H$. pylori group in the current $H$. pylori infection group (adjusted HR: 0.50, 95\% CI: 0.19-1.34, $p=$ 0.17 ) and $H$. pylori eradication group (adjusted HR: 0.51, 95\% CI: $0.18-1.46, p=0.21$ ). In the multivariable Cox models with fixed covariates, HRs and their $95 \%$ CIs of H. pylori eradication for the progression to LSBE remained similar (Supplemental Table 3).

Table 3 Association between RE and H. pylori status pattern and the progression to LSBE adjusted by time-dependent covariate

\begin{tabular}{|c|c|c|c|c|}
\hline & $\mathrm{HR}^{*}$ & \multicolumn{2}{|c|}{$95 \% \mathrm{Cl}$} & $P$-value \\
\hline \multicolumn{5}{|c|}{ RE and H. pylori status pattern } \\
\hline $\mathrm{RE}(-) / H$. pylori $(+)$ & 1.00 & \multicolumn{2}{|c|}{ Reference } & \\
\hline $\mathrm{RE}(-) / H$. pylori $(-)$ & 2.45 & 0.86 & 7.00 & 0.09 \\
\hline $\mathrm{RE}(+) / H$. pylori $(+)$ & 4.57 & 1.26 & 16.63 & 0.02 \\
\hline $\mathrm{RE}(+) / H$. pylori $(-)$ & 7.17 & 2.48 & 20.73 & $<0.001$ \\
\hline Male & 1.18 & 0.41 & 3.45 & 0.76 \\
\hline Age (year) & 1.05 & 1.01 & 1.09 & 0.01 \\
\hline Smoking (100 pack-year) & 3.66 & 1.09 & 12.29 & 0.04 \\
\hline Hiatal hernia & 3.13 & 1.49 & 6.58 & 0.003 \\
\hline PPI or H2RA & 3.99 & 1.54 & 10.33 & 0.004 \\
\hline
\end{tabular}

*Adjusted by time-dependent covariate (RE and H. pylori status pattern, age, smoking, and PPI or H2RA) and baseline time-fixed covariate (male and hiatal hernia)

RE Reflux esophagitis; H. pylori Helicobacter pylori; LSBE Long-segment Barrett's esophagus; HR Hazard ratio; Cl Confidence interval; PPI Proton pump inhibitor; H2RA Histamine H2-receptor antagonist

\section{Discussion}

This is the first retrospective cohort study to investigate the association of $\mathrm{RE}$ and $H$. pylori with the progression from endoscopic SSBE to LSBE referring to changes in RE and $H$. pylori status. Progression to LSBE is comparatively rare in Japanese individuals. RE and non- $H$. pylori infection were associated with a higher rate of progression to LSBE, considering the changes in exposures. H. pylori infection was associated with the prevention of the development of LSBE after the multivariable adjustment for potential confounders, including RE. In addition, the environment preventive of the development of LSBE persists for at least a few years after $H$. pylori eradication.

Time-dependent Cox models may have accurately estimated the relationship between time-varying RE and $H$. pylori status and the progression to LSBE. To date, a few studies have investigated the natural history of endoscopic or histological SSBE [9, 22, 29]. Among them, two studies reported the association between $H$. pylori infection/AG and SSBE elongation within 2- or 5.7-year periods $[9,22]$. One study revealed that shortening of endoscopic SSBE tended to be associated with $H$. pylori and AG, although this was statistically nonsignificant [9]. Another study showed that the absence of AG was associated with the elongation of endoscopic SSBE [22]. These past studies were well designed; however, they did not take into account both the change in RE status and H. pylori eradication. These analyses may have underestimated the association between RE and $H$. pylori status and progression to LSBE. In our analysis, Cox models with baseline time-fixed covariates provided the attenuated HRs of RE and non-H. pylori infection for the progression to LSBE compared to time-dependent Cox models. In addition, the HR of smoking for the progression to LSBE was underestimated in Cox models by baseline time-fixed covariate. These results suggest that time-dependent Cox models were useful to accurately estimate the effects of time-varying exposures such as $\mathrm{RE}, H$. pylori status, and smoking.

$H$. pylori infection was associated with the prevention of the development of LSBE in the time-dependent Cox models. This result is, at least in part, biologically plausible with respect to a decrease in gastric acid secretion. $H$. pylori infection causes gastric atrophy, decreasing 
Table 4 Association between H. pylori status and the progression to LSBE adjusted by time-dependent covariate

\begin{tabular}{|c|c|c|c|c|c|c|c|c|}
\hline & \multicolumn{4}{|c|}{ Multivariable model 1} & \multicolumn{4}{|c|}{ Multivariable model 2} \\
\hline & \multirow[t]{2}{*}{$\mathrm{HR}^{*}$} & \multicolumn{2}{|c|}{$95 \% \mathrm{Cl}$} & \multirow[t]{2}{*}{$P$-value } & \multirow[t]{2}{*}{$\mathrm{HR}^{*}$} & \multicolumn{2}{|c|}{$95 \% \mathrm{Cl}$} & \multirow[t]{2}{*}{$P$-value } \\
\hline \multicolumn{5}{|l|}{ H. pylori status } & & & & \\
\hline Absence of H. pylori & 1.00 & Refer & & & 1.00 & Refer & & \\
\hline H. pylori infection (Model 1) & 0.48 & 0.22 & 1.07 & 0.07 & & & & \\
\hline Current H. pylori infection (Model 2) & & & & & 0.50 & 0.19 & 1.34 & 0.17 \\
\hline H. pylori eradication (Model 2) & & & & & 0.51 & 0.18 & 1.46 & 0.21 \\
\hline Male & 1.19 & 0.41 & 3.47 & 0.75 & 1.19 & 0.41 & 3.47 & 0.75 \\
\hline Age (year) & 1.05 & 1.01 & 1.09 & 0.01 & 1.05 & 1.01 & 1.09 & 0.01 \\
\hline Reflux esophagitis & 3.27 & 1.63 & 6.56 & $<0.001$ & 3.27 & 1.63 & 6.55 & $<0.001$ \\
\hline Smoking (100 pack-year) & 3.67 & 1.10 & 12.26 & 0.03 & 3.67 & 1.10 & 12.29 & 0.03 \\
\hline Hiatal hernia & 3.16 & 1.50 & 6.66 & 0.003 & 3.16 & 1.50 & 6.66 & 0.003 \\
\hline PPI or H2RA & 4.00 & 1.54 & 10.36 & 0.004 & 4.00 & 1.54 & 10.35 & 0.004 \\
\hline
\end{tabular}

*Adjusted by time-dependent covariate (H. pylori status, age, reflux esophagitis, smoking, and PPI or H2RA) and baseline time-fixed covariate (male and hiatal hernia).

H. pylori Helicobacter pylori; LSBE Long-segment Barrett's esophagus; HR Hazard ratio; CI Confidence interval; PPI Proton pump inhibitor; H2RA Histamine H2-receptor antagonist

gastric acid secretion [9]. The decrease in gastric acid secretion prevents RE, leading to the prevention of the development of LSBE. However, our study revealed that $H$. pylori infection was associated with the prevention of the development of LSBE after the multivariable adjustment for potential confounders, including RE. Indeed, the non$\mathrm{RE} / H$. pylori-positive patients were at lower risk of progression to LSBE than the non-RE/H. pylori-negative patients. Although the mechanism by which $H$. pylori infection suppressed the progression to LSBE independent of RE remains unclear, microbiota change in the esophagogastric junction and stomach may affect this suppression of the development of LSBE. Previous studies revealed that $H$. pylori infection reduces the diversity of bacterial microbiome in the stomach [30-32]. Furthermore, a prospective population-based study showed that $H$. pylori infection is involved in gastric microbial dysbiosis, which is associated with carcinogenesis [33]. Although the relationship between microbial dysbiosis and the development of LSBE has not been well studied, microbiota change in the esophagogastric junction and gastric mucosa caused by $H$. pylori infection may create an environment preventive of the development of LSBE.

The environment preventive of the development of LSBE persists for at least a few years after $H$. pylori eradication. Past studies have shown that $H$. pylori eradication increases gastric acid secretion, leading to an increased prevalence of RE [14, 34, 35]. This suggests that $H$. pylori eradication may also increase the rate of endoscopic SSBE elongation. Our study, however, failed to show such a tendency. Our results may indicate that $H$. pylori eradication may not increase the rate of progression to LSBE. However, the results should be interpreted with caution. First, it was uncertain whether the follow- up period was sufficient to assess the association between $H$. pylori eradication and progression to LSBE. Past studies have shown that the risk of developing RE increases as the period after $H$. pylori eradication is extended [35]. Because in many cases, SSBE extends to LSBE after developing RE, H. pylori eradication may promote the progression to LSBE with longer follow-up. Furthermore, the association between $H$. pylori eradication and progression to LSBE may differ depending on the degree of AG before eradication. Only among subjects with mild AG, H. pylori eradication may increase gastric acid secretion, leading to progression to LSBE. Long-term prospective cohort studies are expected that take into account the degree of AG at the time of $\mathrm{H}$. pylori eradication.

The association between the other possible risk factors and progression to LSBE was almost consistent with the reported studies [9, 22, 29]. In our study, age, male sex, smoking, and $\mathrm{HH}$ were associated with the progression to LSBE. However, the relationship between the administration of PPI or H2RA and the progression to LSBE was different from that in the previous study [9]. In our study, the administration of PPI or H2RA was associated with the progression to LSBE. One possible explanation for this is that PPI/H2RA is confounded by severe RE or gastroesophageal reflux disease, which are risk factors of elongation of endoscopic SSBE. That is, subjects taking PPI or H2RA may have potentially severe RE or gastroesophageal reflux disease, resulting in progression to LSBE. However, such an association could not be demonstrated in our database. As our objective for the multivariable adjustment was not to assess the increase in risk with these medications, actively interpreting a coefficient of PPI/H2RA may be misleading [36]. 
Progression from endoscopic SSBE to LSBE is comparatively rare in Japanese individuals. A major reason for the low incidence of developing LSBE may be the different clinical criteria of SSBE. In the United States and Europe, intestinal metaplasia is needed for the diagnosis of SSBE; however, SSBE is diagnosed without histological confirmation of intestinal metaplasia in Japan and the United Kingdom [4, 37, 38]. Endoscopic SSBE in Japan may include more cases that are not diagnosed with $\mathrm{BE}$ according to the criteria of other countries. Indeed, there are far more subjects endoscopically diagnosed with SSBE in Japan than those histologically diagnosed with SSBE in other countries [39]. A substantial proportion of endoscopic SSBE cases in Japan may have lower malignant potential. In fact, in our study, only 34 subjects developed LSBE (1.0 per 1000 personyears). This result suggests that not all subjects with endoscopic SSBE should be followed-up closely. Accurate selection of patients with a high risk for developing LSBE is important, and follow-up of these high-risk patients should be prioritized. Absence of $H$. pylori infection may be a marker of microbiota, which may be associated with a relatively high risk of developing LSBE.

There were several limitations. First, the accurate endoscopic assessment of the length of the $\mathrm{BE}$ is difficult; therefore, its reproducibility is relatively low [40]. To minimize this problem, we evaluated only whether the length of circumferential Barrett epithelium was more than $3 \mathrm{~cm}$ or not (LSBE or SSBE) [29]. Additionally, all cases initially diagnosed with LSBE by endoscopy experts were reconfirmed by the most experienced endoscopy specialist. However, this endoscopic diagnosis of SSBE and LSBE may lead to bias. For example, our analysis may not detect cases with subtle elongation of $\mathrm{BE}$ and may underestimate the prevalence of the development of LSBE. Indeed, among the 7637 subjects with SSBE, only 34 developed LSBE (1.0 per 1000 personyears). Second, the criteria for BE in Japan differ from those in Western countries [37]. At present, the definition of BE has not been universally established. In the United States and most European countries, histological confirmation of intestinal metaplasia is necessary for the diagnosis of $\mathrm{BE}$, unlike in the United Kingdom and Asian countries. In our study, LSBE and SSBE were endoscopically diagnosed without histological examinations using the Prague C \& M criteria. A previous study suggested that endoscopic diagnosis of $\mathrm{BE}$ using the Prague $C$ \& $M$ criteria is relatively reliable, especially in the case of LSBE [40], but differences in the definition of SSBE may affect the results. In fact, a large crosssectional study in Japan showed that the clinical features of endoscopic SSBE differ from those of Western SSBE [8]. Therefore, the results of this study may not be applicable to patients with SSBE worldwide. Third, all subjects in our study voluntarily took these medical surveys. Most subjects were men with medium and high socioeconomic status. Additionally, subjects who did not undergo an additional health-check program after being diagnosed with SSBE were excluded. These may have led to a selection bias. Fourth, the frequency of medical surveys varied from subject to subject. Finally, the number of subjects who experienced the progression to LSBE was rather small. A large long-term study is expected in the future.

\section{Conclusions}

We showed that progression to LSBE is comparatively rare in Japanese individuals. Non-RE and H. pylori infection was associated with a lower rate of progression to LSBE in a Japanese population, considering the changes in exposure. $H$. pylori infection was associated with the prevention of the development of LSBE irrespective of RE. In addition, the environment preventive of the development of LSBE persists for at least a few years after $H$. pylori eradication.

\section{Supplementary information}

Supplementary information accompanies this paper at https://doi.org/10. 1186/s12876-020-01418-5.

Additional file 1 Supplemental Table 1. Subject characteristics at baseline. Supplemental Table 2. Association between RE and H. pylori status and the progression to LSBE adjusted by baseline time-fixed covariate. Supplemental Table 3. Association between H. pylori status and the progression to LSBE adjusted by baseline time-fixed covariate.

\section{Abbreviations}

BE: Barrett's esophagus; EAC: Esophageal adenocarcinoma; SSBE: Shortsegment Barrett's esophagus; LSBE: Long-segment Barrett's esophagus; RE: Reflux esophagitis; H. pylori: Helicobacter pylori; OR: Odds ratio;

Cl: Confidence interval; AG: Atrophic gastritis; PPI: Proton pump inhibitor; H2RA: Histamine H2-receptor antagonist; HRs: Hazard ratios

\section{Acknowledgements}

We thank the clinical technologists and radiological technologists of NTT Medical Center Tokyo for their excellent technical assistance.

\section{Authors' contributions}

Conception and design: GU, TS; Acquisition of data: TJ, KF, TG, NM; Analysis and Interpretation of data: GU, TS; Drafting of the manuscript: GU; Critical revision of the manuscript for important intellectual content: TS, TM, NM; Statistical analysis: GU, TS; Supervision: TM, TG, NM. The author (s) read and approved the final manuscript.

\section{Funding}

Not applicable.

\section{Availability of data and materials}

The datasets used and/or analyzed during the current study are available from the corresponding author on reasonable request.

Ethics approval and consent to participate

The study protocol was approved by the local institutional ethics review board (ethics committee of NTT Medical Center, Tokyo).

Consent for publication

Not applicable. 


\section{Competing interests}

The authors declare that they have no competing interests.

\section{Author details}

'Department of Diagnostic Pathology, NTT Medical Center Tokyo, 5-9-22 Higashi-gotanda, Shinagawa-ku, Tokyo 141-8625, Japan. ${ }^{2}$ Department of Information and Computer Technology, Faculty of Engineering, Tokyo University of Science, Tokyo, Japan. ${ }^{3}$ Center for Preventive Medicine, NTT Medical Center, Tokyo, Tokyo, Japan. ${ }^{4}$ Department of General Medicine, Juntendo University Hospital, Tokyo, Japan. ${ }^{5}$ Department of Gastroenterology, NTT Medical Center, Tokyo, Tokyo, Japan.

Received: 9 April 2020 Accepted: 9 August 2020

Published online: 15 August 2020

\section{References}

1. Spechler SJ. Barrett esophagus and risk of esophageal cancer: a clinical review. JAMA. 2013:310:627-36.

2. Spechler SJ, Souza RF. Barrett's esophagus. N Engl J Med. 2014;371:836-45

3. de Jonge PJ, van Blankenstein M, Grady WM, et al. Barrett's oesophagus: epidemiology, cancer risk and implications for management. Gut. 2014;63: 191-202.

4. Sharma P, Morales TG, Sampliner RE. Short segment Barrett's esophagus - the need for standardization of the definition and of endoscopic criteria. Am J Gastroenterol. 1998;93:1033-6.

5. Hamade $N$, Vennelaganti S, Parasa S, et al. Lower annual rate of progression of short-segment vs long-segment Barrett's esophagus to esophageal adenocarcinoma. Clin Gastroenterol Hepatol. 2019;17:864-8.

6. Chang CY, Cook MB, Lee YC, et al. Current status of Barrett's esophagus research in Asia. J Gastroenterol Hepatol. 2011:26:240-6.

7. Akiyama T, Inamori M, Akimoto $K$, et al. Risk factors for the progression of endoscopic Barrett's epithelium in Japan: a multivariate analysis based on the Prague C \& M criteria. Dig Dis Sci. 2009;54:1702-7.

8. Usui G, Sato H, Shinozaki T, et al. Association between helicobacter pylori infection and short-segment/long-segment Barrett's esophagus in a Japanese population: a large cross-sectional study. J Clin Gastroenterol. 2020:54:439-4.

9. Okita K, Amano Y, Takahashi Y, et al. Barrett's esophagus in Japanese patients: its prevalence, form, and elongation. J Gastroenterol. 2008:43:92834.

10. Kuipers EJ, Uyterlinde AM, Pena AS, et al. Long-term sequelae of helicobacter pylori gastritis. Lancet. 1995;345:1525-8.

11. El-Omar EM, Oien K, El-Nujumi A, et al. Helicobacter pylori infection and chronic gastric acid hyposecretion. Gastroenterology. 1997;113:15-24.

12. Lee YC, Chiang TH, Chou CK, et al. Association between Helicobacter pylori eradication and gastric cancer incidence: a systematic review and metaanalysis. Gastroenterology. 2016;150:1113-24.e5.

13. Choi IJ, Kook MC, Kim Yl, et al. Helicobacter pylori therapy for the prevention of metachronous gastric cancer. N Engl J Med. 2018;378:108595.

14. Nam SY, Choi IJ, Ryu KH, et al. Effect of helicobacter pylori infection and its eradication on reflux esophagitis and reflux symptoms. Am J Gastroenterol. 2010;105:2153-62.

15. O'Connor A, O'Morain CA, Ford AC. Population screening and treatment of helicobacter pylori infection. Nat Rev Gastroenterol Hepatol. 2017;14:230-40

16. Usui $G$, Shinozaki $T$, Jinno $T$, et al. Association between visceral abdominal obesity and long-segment Barrett's esophagus in a Japanese population. J Gastroenterol. 2020:55:189-97.

17. Gunji T, Matsuhashi N, Sato H, et al. Helicobacter pylori infection is significantly associated with metabolic syndrome in the Japanese population. Am J Gastroenterol. 2008;103:3005-10.

18. Lundell LR, Dent J, Bennett JR, et al. Endoscopic assessment of oesophagitis: clinical and functional correlates and further validation of the Los Angeles classification. Gut. 1999:45:172-80.

19. Dent J. Endoscopic grading of reflux oesophagitis: the past, present and future. Best Pract Res Clin Gastroenterol. 2008;22:585-99.

20. Sharma P, Dent J, Armstrong D, et al. The development and validation of an endoscopic grading system for Barrett's esophagus: the Prague C \& M criteria. Gastroenterology. 2006;131:1392-9.
21. Matsuhashi N, Sakai E, Ohata K, et al. Surveillance of patients with longsegment Barrett's esophagus: a multicenter prospective cohort study in Japan. J Gastroenterol Hepatol. 2017;32:409-14.

22. Manabe N, Haruma K, Imamura $\mathrm{H}$, et al. Does short-segment columnar-lined esophagus elongate during a mean follow-up period of 5.7 years? Dig Endosc. 2011;23:166-72

23. Shimoyama S, Ogawa T, Toma T, et al. A substantial incidence of silent short segment endoscopically suspected esophageal metaplasia in an adult Japanese primary care practice. World J Gastrointest Endosc. 2012;4:38-44.

24. Shimoyama S, Ogawa T, Toma T. Trajectories of endoscopic Barrett esophagus: chronological changes in a community-based cohort. World J Gastroenterol. 2016;22:8060-6.

25. Matsuzaki J, Suzuki H, Asakura K, et al. Etiological difference between ultrashort- and short-segment Barrett's esophagus. J Gastroenterol. 2011;46: 332-8.

26. Woodward M, Morrison C, McColl K. An investigation into factors associated with helicobacter pylori infection. J Clin Epidemiol. 2000:53:175-81.

27. Longo-Mbenza B, Nsenga JN, Ngoma DV. Prevention of the metabolic syndrome insulin resistance and the atherosclerotic diseases in Africans infected by helicobacter pylori infection and treated by antibiotics. Int $J$ Cardiol. 2007;121:229-38

28. Kanda Y. Investigation of the freely available easy-to-use software 'EZR' for medical statistics. Bone Marrow Transplant. 2013;48:452-8.

29. Asayama M, Shibata M, Kondo Y, et al. Retrospective cohort study of chronological change of short-segment barrett's esophagus. Dig Endosc. 2005;17:28-31

30. Parsons BN, ljaz UZ, D'Amore R, et al. Comparison of the human gastric microbiota in hypochlorhydric states arising as a result of helicobacter pylori-induced atrophic gastritis, autoimmune atrophic gastritis and proton pump inhibitor use. PLoS Pathog. 2017;13:e1006653.

31. Klymiuk I, Bilgilier C, Stadlmann A, et al. The human gastric microbiome is predicated upon infection with helicobacter pylori. Front Microbiol. 2017;8: 2508 .

32. Schulz C, Schütte $K$, Koch $N$, et al. The active bacterial assemblages of the upper $\mathrm{Gl}$ tract in individuals with and without helicobacter infection. Gut. 2018;67:216-25

33. Guo $Y$, Zhang $Y$, Gerhard $M$, et al. Effect of helicobacter pylori on gastrointestinal microbiota: a population-based study in Linqu, a high-risk area of gastric cancer. 2020;69:1598-607.

34. Sugimoto $M$, Uotani $T$, Ichikawa $H$, et al. Gastroesophageal reflux disease in time covering eradication for all patients infected with helicobacter pylori in Japan. Digestion. 2016;93:24-31.

35. Adachi K, Notsu T, Mishiro T, et al. Long-term effect of helicobacter pylori eradication on prevalence of reflux esophagitis. J Gastroenterol Hepatol. 2019;34:1963-7.

36. Westreich D, Greenland S. The table 2 fallacy: presenting and interpreting confounder and modifier coefficients. Am J Epidemiol. 2013;177:292-8.

37. Shaheen NJ, Falk GW, lyer PG, et al. ACG clinical guideline: diagnosis and Management of Barrett's esophagus. Am J Gastroenterol. 2016;111:30-51.

38. Salimian KJ, Waters KM, Eze O, et al. Definition of Barrett esophagus in the United States: support for retention of a requirement for goblet cells. Am J Surg Pathol. 2018;42:264-8.

39. Lee HS, Jeon SW. Barrett esophagus in Asia: same disease with different pattern. Clin Endosc. 2014;47:15-22

40. Lee $Y C$, Cook MB, Bhatia S, et al. Interobserver reliability in the endoscopic diagnosis and grading of Barrett's esophagus: an Asian multinational study. Endoscopy. 2010:42:699-704.

\section{Publisher's Note}

Springer Nature remains neutral with regard to jurisdictional claims in published maps and institutional affiliations. 